International Journal of Linguistics, Literature and Translation

ISSN: 2617-0299 (Online); ISSN: 2708-0099 (Print)

DOI: $10.32996 / \mathrm{ijllt}$

Journal Homepage: www.al-kindipublisher.com/index.php/ijllt

IJLLT

\title{
Investigating the Persuasive Writing Performance of Moroccan Advanced EFL Students: Is it a problem of "Language" or 'Reasoning" Acquisition Device?
}

\author{
Brahim Khartite ${ }^{1}$ 8(D), Bendaoud Nadif ${ }^{2}$ 8 (D) $\triangle$ and Ismail Benfilali ${ }^{3}$ (iD \\ ${ }^{1}$ Assistant Professor of Engineering/Business English ENSAM, Meknes Moulay Ismail University, Morocco \\ ${ }^{2}$ Ph.D. Candidate, English Department, Moulay Ismail University, Meknes, Morocco \\ ${ }^{3}$ Assistant Professor, English Department, Sidi Mohamed Ben Abdellah University, Fez-Sais, Morocco \\ $\triangle$ Corresponding Author: Bendaoud Nadif, E-mail: bendaoudnadif@gmail.com
}

\section{ARTICLE INFORMATION}

Received: April 08, 2021

Accepted: May 11, 2021

Volume: 4

Issue: 5

DOI: $10.32996 /$ ijllt.2021.4.5.6

\section{KEYWORDS}

Contrastive rhetoric, Moroccan EFL writing transfer, persuasive essays, reasoning strategies, rhetorical patterns

\section{ABSTRACT}

This study investigates the extent to which the results of rhetorical comparisons of persuasive essays by US English native speakers and others by Moroccan advanced EFL students will provide empirical evidence for Kaplan's (1966) contrastive rhetoric hypothesis. This is especially regarding the fact that EFL students-writing problems are a byproduct of the negative transfer of rhetorical strategies from their first language (L1). This hypothesis is tested by comparing $20 \mathrm{EFL}$ and Arabic L1 persuasive essays by the same EFL students to essays in English as L1 by native speakers to identify the extent to which the language of composing and one's cultural background affects the writing quality of their essays. The study hypothesizes that if Kaplan's contrastive rhetoric claims were accurate, then Moroccan advanced EFL writers would produce essays that tend to be rhetorically less accurate when judged by standard English rhetorical criteria. Moreno's (2005) approach to match comparable corpora of persuasive essays from two different cultural and linguistic backgrounds was adopted. As for the study participants, 40 advanced student-writers from two discrepant language and cultural backgrounds were recruited to take part in the study. While the results of a stepwise multiple regression analysis provides further evidence corroborating the validity of the rhetorical measures used in the study, group mean scores comparisons and a Multiple Discriminant analysis of the data indicates that those writers from various cultural backgrounds seem to face far more similar than different rhetorical problems and their writing inadequacies are equally distributed regardless of which language the study participants used to write their essays.

\section{Introduction}

Composing in a language other than one's first is a very complex process. There seems to be a general agreement among ELT practitioners that out of all the four skills related to second language learning and or acquisition, writing is described as the most challenging not only for teachers to teach but also for students to learn and acquire. In fact, it is challenging to write in one's first language, let alone in a foreign or second language. It has also been observed that experienced teacher of writing and composition can effortlessly spot writing by non-native speakers even when the latter's pieces of writing are free of grammatical, lexical and syntactic inaccuracies. This article attempts to identify if the main assumptions of the Contrastive rhetoric hypothesis by Kaplan (1966) might be used to account for "the clumsiness" or "foreignness" phenomenon underlying the persuasive writing challenges of advanced EFL students in the Moroccan context.

\section{Statement of the Problem}

The problem underlying the teaching of writing and composition in the Moroccan context is that both teachers and students are quite dissatisfied. Teachers tend to feel generally discontented when it comes to evaluating the quality of their EFL students' writing assignments, even at advanced language proficiency level. Similarly, advanced EFL students, in their turn, constantly complain

K C AL-KINDI CENTER

$R$ DFOR RESEARCH AND DEVELOPMENT

Your gateway to world-class research

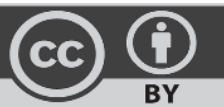

Published by Al-Kindi Center for Research and Development, London, United Kingdom. Copyright (c) the author(s). This open access article is distributed under a Creative Commons Attribution (CC-BY) 4.0 license 
about the frustrating feedback comments they receive from their writing instructors and supervisors who tend to describe their writing assignments as "clumsy"; "awkward and incoherent" or "repetitive and redundant". Students get confused, especially when these comments are used to describe or evaluate these pieces of writing that apparently look free of structural inaccuracies and or grammatical mistakes. The aims of the present article is to address the persuasive writing performance of Moroccan advanced EFL students to explain the increasing dissatisfaction of both teachers and students when it comes to evaluating the quality of their writing assignments in an EFL context like Morocco. Put more specifically, this paper attempts to examine the validity of Kaplan's hypothesis that Moroccan advanced EFL students face writing challenges as a result of L1 rhetorical transfer. This will be achieved by examining the extent to which it is true that the persuasive essays of two groups of EL1 and EFL advanced students from two cultural and linguistic backgrounds are rhetorically dissimilar as a result of one's L1 interference.

\section{Background of the Study}

Kaplan (1966) and his supporters has long ago tried to account for the clumsiness of writing by advanced ESL and EFL writers. He claims that the sense of 'foreignness' underlying essays by non-native writers and the above-mentioned feedback comments by writing instructors are "essentially accurate because when writing in a second language, foreign students tend to employ a rhetoric or a sequence of thought that violates the English native speakers expectations of how ideas are arranged and how topics are developed"(Kaplan 1966:16) As far back as (1966) Kaplan attempted to account for this problem by introducing the contrastive rhetoric hypothesis claiming that "all along their acquisition of their first language children also learn acceptable forms of reasoning and acceptable forms of sequencing their thought patterns" (p.14). In other words, he seems to imply that in much the same way there is a critical hypothesis period for language acquisition (LAD or language acquisition device), there is also a similar critical period that applies to the thought patterns and reasoning strategies called (RAD or reasoning acquisition device). This implies that once one learns how to reason in a first language will make reasoning in a second language very challenging, if not impossible. Obviously, given the fact that reasoning and logic are cultural and language bound, Koch, one of the supporters of Kaplan's hypothesis, maintains that when these students set out to write in the new context of a second or foreign language, they have a tendency to make "high order thinking or global mistakes in how ideas are arranged and how the topic is approached and developed" (Koch 1881: 02) because the logic and rhetoric underlying their writing is usually deeply rooted in their L1 and culture. Hence, Kaplan's fundamental claim that EFL writing problems of non-native speakers are often than not a by-product of negative transfer of rhetorical patterns from their L1 and culture.

Kaplan based his hypothesis on the assumptions of two previous hypotheses prevalent at the time of his study. The first is SapirWorlf hypothesis -also known as the linguistic relativity hypothesis or deterministic theory (1929). It posits that the language one speaks shapes the way they think, determines their perception and eventually influences their behaviour. The core theory seems especially to underline that discourse, logic and thought patterns are all interrelated and tend to feed into each other in a very elusive and subtle way. What is common among all of these elements, however, is that they are culture and language-specific.

Kaplan extends the above assumptions into the ESL context and concludes that what is described as a good piece of writing or composition is usually a culturally defined concept. This is probably the reason why the way non-native users of English structure discourse is said to be shaped and sometimes determined by their first language and culture; the way foreign language learners in general write and structure their discourse is broadly influenced by the way they think and reason, both of which processes are deeply rooted in their first language and/or culture (RAD).

The second hypothesis upon which Kaplan based his assumptions is the contrastive analysis theory, also known as the negative language transfer hypothesis by Fries (1945), Lado (1957). This hypothesis is based on the assumption that syntactic and grammatical errors in a second language class are mostly attributable to negative transfer from the students' first language. Therefore, the wider the gap and the bigger the differences between one's L1 and the second or foreign language one is trying to learn, the more challenging they will find it to learn and be able to reach a native-like mastery using the target language as a written medium.

Accordingly, Kaplan combined the assumptions of both hypotheses and came up with his own hypothesis, generally known as the contrastive rhetoric hypothesis and recently referred to as intercultural rhetoric hypothesis but famously associated in the literature of writing and composition with the following figure/diagrams. 


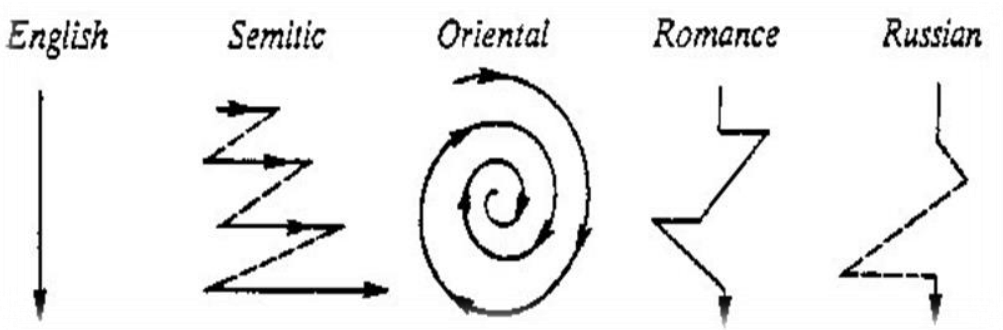

Language Learning: Cultural Thought Patterns in Intercultural Education.

(Kaplan 1966: p.14)

Upon analysing more than 600 ESL paragraphs by ESL students originating from various language backgrounds, Kaplan draws attention to the idea that writing in English is typically direct and vertical; its logic or sequence of thought is mostly linear and deductive. This is in sharp contrast to some other languages (including Arabic) that tend to be poetic, repetitive, digressive, horizontal and their logic is rather inductive or spiral (Koch 1983). Kaplan also observes that, unlike Arabic, where much emphasis is put on the form and the beauty of the language, English emphasises the meaning and logical reasoning instead.

After Kaplan's (1966) study, a great many rhetoricians hastened to provide further empirical evidence in favour of its underlying assumptions. For example, Koch (1981) observes that there is a tendency to overuse clichés, formulaic expressions or proverbial saying in Arabic written discourse. There is also a tendency towards use of anecdotal reasoning and arguing by analogy or repetition in a discourse that is overloaded with coordinate clauses and parallel structures. In English, however, use of logical reasoning, originality and linear development of ideas together with creative thought and conciseness in a discourse that favours deletion and subordination are all encouraged and recommended in writing and composition manuals.

\section{The study}

Unfortunately, although Kaplan's assumptions are not yet empirically substantiated, many ESL writing teachers do still share his diagrams and some of the above-mentioned rhetorical differences and sweeping generalisations about various languages and cultures with their students as facts that are beyond any shadow of a doubt. Also, some textbook writers and writing-manual designers at various language proficiency levels use them despite the absence of any empirical evidence in favour of all those alleged rhetorical differences. Some ESL teachers - including the authors of the current article - are quick to explain to their students that there is no way they could write as fluently or as eloquently as native speakers simply because of their L1 transfer. As a matter of fact, the authors of the current paper had taught writing with the same mind-set for a good while before they grew sceptic and decided to run the current empirical study to check the validity of Kaplan's hypothesis in the Moroccan context. In fact, this paper assumes that using the diagrams above in EFL writing classes prior to checking their validity might only be perpetuating Kaplan's speculative ideas and unsubstantiated assumptions about different languages and/or cultures.

\subsection{Objectives}

The current study has two main objectives: first, it seeks to explore the validity of Kaplan's (1966) fundamental claim that thought patterns governing the development of persuasive writing are cultural or language specific. Second, it attempts to find out if the writing problems of Moroccan advanced EFL students are truly attributable to their L1 interference at the rhetorical level.

\subsection{Methodology}

The research design of the study was engineered, bearing in mind some of the major theoretical flaws underlying early contrastive rhetoric studies. More specifically, Moreno's (2005) approach to match comparable corpora of persuasive essays from two different cultural and linguistic backgrounds was adopted. As for the study participants, 40 advanced student-writers from two discrepant language and cultural backgrounds were recruited to take part in the study. For this purpose, a convenience sampling for the Moroccan EFL group and a snowball sampling procedure for EL1 participants are used. Only 20 advanced Moroccan ELF advanced students and 20 USA EL1 participants are eventually involved in the study. While the same Moroccan group writes persuasive essays - spaced out in time for validity reasons- in both Arabic and English, the EL1 group responded to the same writing task only in their native language (i.e. English). Prior to the main study, participants are required to fill in a language history questionnaire for the study to ascertain that the requirements of an adequate tertuim comparationis are established (Tertium comparationis is Latin for "the third [part] of the comparison". It is the quality that two things which are being compared have in common) before any cross-cultural or cross-linguistic comparisons are conducted.

It is not without relevance also to underline that the current study adopts a descriptive and non-judgmental instead of prescriptive and value-laden approach to the examination of rhetorical patterns of both study participants from two language and cultural backgrounds. This means that an equal status of power is assigned to the two languages/cultures and subjects under study. The 
study attempts to elicit comparable writing corpora without eliminating or marginalizing ESL students' rhetorical preferences as irrelevant. This implies that the main purpose of the study is not to find out which study group will write better or more adequate persuasive essays. It will simply find out if Moroccan advanced EFL students and their US EL1 counterparts face similar or different rhetorical problems that result from one's L1 transfer when developing their persuasive essays.

Equally important is the fact that care is especially taken to avoid falling into the trap of methodology flaws of some previous studies like speculating about students' L1 rhetorical patterns by studying their EFL or ESL writing; comparing student writing to professional/published writing, comparing beginner ESL writing to advanced English L1 writing, and finally a tendency to use intuition or invalid analytic measures to conduct cross-cultural and cross-linguistic rhetorical comparisons.

The current study uses valid analytic tools adapted from Connor and Lauer (1985) to measure participants' persuasive performance. Therefore, the rhetorical patterns used are well defined and their validity is theoretically established. The data tabulated from the language history questionnaire revealed that none of the subjects involved in the study failed to meet the requirement of an adequate tertium comparationis, especially with regards to such variables as age, proficiency level, major of study and cognitive maturity.

\subsection{Research hypotheses}

For Kaplan's contrastive rhetoric claims attributing writing problems of advanced EFL students to their L1 transfer to be accepted as true and valid, the following hypotheses must be confirmed.

1. Participants' Rhetorical performance will correlate significantly with their overall writing performance.

2. There are no significant differences in the rhetorical performance of EFL and AL1 of advanced Moroccan writers on the same persuasive task.

3. The rhetorical performance of all the study participants will accurately predict their $\mathrm{L} 1$ and/or cultural background.

4. There are significant differences in the rhetorical performance of US EL1 and that of their Moroccan advanced EFL counterparts.

5. The rhetorical problems of Moroccan advanced EFL writers, if any, are a result of their L1 transfer.

The first research hypothesis is meant to check and thus establish the validity of the analytic measures/rhetorical patterns in the study. For this purpose, the study resorts to the use of A Computerized Stepwise Multiple-Regression analysis as a statistical measure wherein the criterion variable in the regression model was specified as holistic scores and the predictor variables were the analytic tools; namely argument superstructure, Toulmin's informal reasoning, and persuasive appeals. In fact, a Stepwise Regression Analysis is used thanks to its ability to calculate the predictive power of each analytic tool per se or in a combination of two or more variables at the same time.

The other four hypotheses are geared to test the assumptions of Kaplan's contrastive rhetoric hypothesis as it applies to the persuasive essays of Moroccan advanced EFL students. More precisely, the second hypothesis assumes the existence of no rhetorical differences in the persuasive essays of the same Moroccan study participants - because their essays are presumably produced by a similar cultural logic deeply rooted in their L1 and or culture. The third hypothesis uses a Multiple Discriminant statistical measure to check the impact of one's L1 or culture, if any, on their rhetorical performance. The fourth research hypothesis assumes essays written by the two study groups to be different again because, judged from Kaplan's (1966) same line of reasoning, they are produced by a different cultural Logic. The last research hypothesis is geared to unveil if it is true as Kaplan (1966) claims that Moroccan EFL advanced students tend to transfer their L1 patterns to the new context of English as a foreign language. For this purpose, ANOVA is expected to confirm that the mean square error (within group differences) is significantly lower than the mean square effect (between group differences) as a result of the students' L1 transfer.

\section{Results of the study}

The results reveal that the mean scores of the participant' overall writing performance (as measured by the holistic scores) correlated significantly with the mean scores of their rhetorical performance (as measured by the analytical patterns validated in the study). This seems to confirm the first research hypothesis that is specifically geared to establish the validity of the analytic patterns investigated prior to any cross-cultural comparisons. Having subjected the data to a computerized Multiple Regression Analysis to identify the predictive power of the rhetorical variables, it turns out that performance of all the study participants on three measures of rhetorical dimensions, namely data, argument-superstructure and rational-appeals proved capable of predicting the writers' overall writing performance -as measured by the holistic scores- with $78 \%$ degree of accuracy. In their study, Bouih, Nadif and Benattabou (2021), the correlational analysis examining the link between self-efficacy and academic achievement showed a mild positive relationship in favour of composition. In another study, Nadif and Benattabou (2021) reported that good language learners' scores significantly correlated with their achievement scores in reading and language more than writing. 
Interestingly enough, these preliminary findings seem also to partly support one of the basic assumptions of the CR hypothesis that Moroccan EFL writers exhibit signs of similar rhetorical challenges in both their EFL and AL1 persuasive writing, as evidenced by their below-average mean scores $(\mathrm{H} 2)$. It seems, however, that these very same results appear to indicate that, like the Moroccan EFL group, US EL1 group also displays signs of similarly inadequate rhetorical performance. In other words, the rhetorical challenges detected are not exclusively limited to the Moroccan EFL group. In fact, US EL1 group also displays similar poor rhetorical performance or inadequate rhetorical -performance as evidenced by their below-average mean scores both in terms of their overall writing scores and their rhetorical performance.

\subsection{Testing the Contrastive Rhetoric Hypothesis}

For the study to test the fundamental assumptions of Kaplan's contrastive rhetoric hypothesis, both analytic and holistic scores were subjected to a Multiple Discriminant Analysis (MDA). This statistical tool is used as it serves to identify if the rhetorical performance of the study participants can accurately discriminate among the three data sets defined in terms of the language of composing. More specifically, it is hoped to unveil the effect of participants' L1 or culture, if any, on their rhetorical performance. That said, MDA results failed to produce any model that could significantly discriminate among the three data sets defined in terms of the language of composing $g$ ( $A L 1, E F L$ and EL1). This is interpreted as implying that the participants $L 1$ or cultural background do not seem to have impacted the rhetorical performance of the two study groups in any significant way. This amounts to also saying that the writing challenges detected in the study appear to be similarly distributed across to the three types of essays and are therefore not limited to the essays by Moroccan EFL participants. Besides, because ANOVA reveals that withingroup differences (AL1 and EFL) turned out to be higher than the between-group, this suggests that the rhetorical inadequacies underlying the rhetorical performance of the Moroccan advanced EFL students do not seem to result from their first language transfer as claimed by the Kaplan (1966) and/ or his subsequent supporters or advocates of the contrastive rhetoric hypothesis.

\subsection{Major Findings of the Study}

The results on MRA, MDA and ANOVA of the current study are all interpreted as implying that EL1 rhetorical performance of US native English speakers does not seem to be of significantly any better quality than that of Moroccan Advanced EFL writers, at least as far as persuasive essays are concerned. Contrarily to the main assumptions of the $\mathrm{CRH}$, the results of the current study indicate that the rhetorical patterns of English persuasive writing investigated in the study do not seem to be significantly any more problematic for the Moroccan Advanced EFL writers than for their native English speakers' counterparts regardless of the language in which Moroccan EFL student-writers composed their essays. The results also clearly indicate that Kaplan's fundamental claims with regards to L1 rhetorical transfer are not valid. In fact, it seems that both advanced EFL students writing in an EFL context like Moroccan and their US EL1 counterparts writing in English as their L1 do face far more similar than different rhetorical challenges as least as far as writing persuasive essays are concerned.

\section{Implications}

It has been the aim of the current study to explore the validity of Kaplan's (1966) fundamental claim that thought patterns governing the development of persuasive writing are cultural and/or language-specific. The second objective is to find out if the writing problems of Moroccan advanced EFL students are truly attributable to their L1 interference at the rhetorical level. The final conclusions underlying the current study seem thus to go hand in glove with some previous studies it replicates and are quick to cast serious doubts on the fundamental claims of the contrastive rhetoric hypothesis. This eventually amounts to saying that from a research methodological perspective, the current study attests to the fact once all the research design flaws of some previous studies are avoided, (i.e. once all the variables are controlled for and the assumptions of a common grounds for comparable corpora are established but above all once the analytic measures used for cross-cultural comparisons are made specific and well defined) the results of cross-cultural rhetorical comparisons from an intercultural perspective reveal that writers from various cultural backgrounds seem to face far more similar than different rhetorical problems and their writing inadequacies are equally distributed regardless of the language in which participants write their essays. Therefore, the tendency to implicate $L 1$ interference as the main source of writing problems of Moroccan advanced EFL students- does not seem to be supported by the current study and it requires, therefore, further substantial and empirical research evidence. Until then, stigmatizing ESL/EFL writing by nonnative English speakers as less persuasive or rhetorically inappropriate as a result of their L1 interference should be taken with a pinch of salt and must be dealt with cautiously.

If it is generally considered in Applied Linguistics as self-evident that no research paper is devoid of limitations, the present research study is obviously no exception. One of the straightforward limitations underlying the present study consists particularly in the limited number of subjects who volunteered to take part in it. Equally important is that the study failed to conduct any post-writing structured interviews to delve deeper into the participants' rhetorical challenges. Also related to the limitations underlying the current study is the fact that academic level and proficiency levels are used synonymously. The researchers assume that the academic level of the study participants is an accurate indicator of their language proficiency level. This, however, is obviously not always necessarily the case. Future research might probably want to use a standardised test to specify participants' true language proficiency level and this surely yield more insightful and valid results. 


\section{Conclusion}

Put in a nutshell, despite all the limitations underlying the current study, its results and key findings seem to indicate that Kaplan's fundamental claims with regards to L1 rhetorical transfer in the Moroccan context do not appear to be valid. Both Moroccan advanced EFL students and their US EL1 counterparts seem to face similar writing challenges, at least as far as writing persuasive essays are concerned. This eventually amounts to saying that EL1 and EFL students' writing inadequacies are equally distributed regardless of the language in which all the study participants write their essays. The tendency to implicate L1 interference as the main source of writing problems of Moroccan advanced EFL students- does not seem to be supported by the current study. Therefore, stigmatizing EFL writing by non-native English speakers as less persuasive or rhetorically inappropriate as a result of their L1 interference should be dealt with cautiously and this is at least until further substantial and more empirical research evidence is provided.

Funding: This research received no external funding

Conflicts of Interest: The authors declare no conflict of interest.

Acknowledgement: The authors of the current paper would like to acknowledge the valuable contribution of two associate professors- namely Pr. Badiaa Zerhouni- Faculty of Education Rabat and Pr. Naima Trimasse University Ibno Zohr-Agadir- to this study, especially at the stage of collecting data and writing various drafts that brought the paper to its final shape.

\section{About Authors}

1-Brahim Khartite is a PhD holder from the Faculty of Sciences Rabat, Morocco. He is currently an Assistant Professor of ESP and Business English at ENSAM Meknés, Moulay Ismail University Morocco. He is also a PCELT holder and UME alumni in Boston USA (2005) and Seville Spain (2006). His main educational concerns and interests include, among others, schema theory, reading comprehension, second language writing and teacher training and teacher CPD

2-Bendaoud Nadif is a PhD candidate at the Department of English, Faculty of Arts and Humanities, Meknés, Morocco. His research interests revolve around such areas as Applied Linguistics, EFL learning and teaching, and Good Language Learner Research.

3- Ismail Benfilali is a PhD holder and Assistant Professor from the English Department of Sidi Mohamed Ben Abdellah University, Fez-Sais, Morocco. His main research interests are applied linguistics, gender issues and sociolinguistics.

\section{References}

[1] Bouih, A., Nadif, B., \& Benattabou, D. (2021). Assessing the Effect of General Self-efficacy on Academic Achievement Using Path Analysis: A Preliminary Study. Journal of English Language Teaching and Applied Linguistics, 3(4), 18-24.

[2] Connor, U., \& Lauer, J. (1988). Cross-cultural variation in persuasive student writing. In C. Purves (Ed.), Writing across languages and cultures (pp. 138-159). Sage.

[3] Fries, C. (1945).Teaching and learning English as a foreign language. Ann Arbor

[4] Kaplan, R. B. (1966). Cultural thought patterns in inter-cultural education. Language, 16,1-20.

[5] Koch, B. (1981). Repetition in Discourse: Cohesion and Persuasion in Arabic Argumentative Prose. Dissertation Abstracts International, 42(09), 3983A.

[6] Koch, B. (1983c). Presentation as proof: The language of Arabic rhetoric. Anthropological Linguistics, 25(1), 47-60.

[7] Khartite, B., \& Zerhouni, B. (2016). Second Language Writing from an Intercultural Rhetoric Perspective. Arab World English Journal, 7(3). DOl:https://dx.doi.org/10.24093/awej/vol7no3.7. Retrieved 2019-03-15

[8] Khartite, B., \& Zerhouni, B. (2018). A Study into the Writing Performance of Moroccan Advanced EFL Writers from an Intercultural Rhetoric Perspective. Arab World English Journal, 9(3), 390- 405.

i. DOl:https://dx.doi.org/10.24093/awej/vol9no3.26 Retrieved 2020-01-15

[9] Lado, R. (1957). Linguistics Across Cultures: Applied Linguistics for Language Teachers. Ann Arbor

[10] .Lauer, J. M., Montague, G., Lunsford, A., \&Emig, J. (1985). Four worlds of writing (2nd ed.).

[11] Moreno, A. (2005). The importance of comparable corpora in cross cultural studies. In U. Connor, E. Nagelhout, \& W. Rozycki (Eds.), Contrastive rhetoric: Reaching to Intercultural Rhetoric (pp. 25-41). 